\title{
Evaluation and Refinement of Emergency Situation Ontology
}

\author{
Sarika Jain and Valerie Meyer
}

\begin{abstract}
Ontologies provide a machine-processable description of entities and their properties, relationships and constraints, thus they can depict the semantics of disaster situations and related emergency tasks and thereby help to create connections between them for an efficient emergency response. As an ontology acts as the basic structure and knowledge base of an application, evaluation and assessment of the ontology are a critical point of the development process. Through the evaluation, the quality and the content of the ontology is assessed and it ensures that the ontology is well built, structured and contains all important concepts and relationships between them for sufficient reasoning. In this paper, an evaluation framework is proposed to evaluate an emergency situation ontology for which existing evaluation methods have been combined into a single framework, dividing the methods used into two phases: verification and validation. The verification of the ontology ensures that the ontology is correctly built. It evaluates the structure, functionality and representation of the ontology. Different metrics and common pitfalls are used to detect errors. The validation of the ontology ensures that the right ontology for the given application is built. This is achieved by competency questions and expert interviews.
\end{abstract}

Index Terms-Evaluation framework, emergency situation, ontology, verification, validation.

\section{INTRODUCTION}

Web 1.0 is read only, e.g., shopping carts; Web 2.0 is read and write facilitating interaction, e.g., social networks; Web3.0 is read write and execute facilitating intelligence. Web 3.0 or simply semantic web is the web of the future which helps machines understand the web page and makes life easier. The semantic web is able to integrate and combine the data drawn from diverse sources because of the availability of common formats and meta data in the form of ontology. An ontology is a fundamental data structure for conceptualizing knowledge. Ontologies capture background knowledge by providing structured information in means of relevant concepts, properties and their relations in a machine-processable way. They enable us formalize knowledge of a certain domain and therefore enable automatic gathering of data and reasoning on them [1]. Design and development of an ontology is a complex and ambiguous task, which takes a lot of effort and needs to be constantly reviewed to judge if concepts and relationships included are correct [2]. This paper aims to assess the quality

Manuscript received October 31, 2017; revised May 14, 2018.

S. Jain is with the National Institute of Technology, Kurukshetra, India (e-mail: jasarika@ nitkkr.ac.in).

V. Meyer is with the Universitaet of Osnabrueck, Osnabrueck, Germany (e-mail: vameyer@uni-osnabrueck.de). and the content of the Emergency Situation Ontology (ESO) to ensure that the ontology is well built, structured and contains all important concepts and relationships for sufficient reasoning. The ontology evaluated in this study, ESO, has been developed to provide recommendations and expert advice in immediate emergency response.

There is a requirement of creating citywide situational awareness and its emergency management. For providing prompt decisions during disaster management, the city personnel/planners need to focus upon the effective and efficient storage, analysis and processing of emergency information, safety plans and resources to generate the recommendations along with proper justification [3]. In recent years, ontology tools have been widely used for representation and reasoning in situations of emergency [4], [5]. It builds the knowledge base for an intelligent decision support system which enables an automatic and effective response to all kinds of disasters. The aim of this decision support system is to minimize the loss of life and property, but its best results can only be guaranteed if high-quality ontology is provided [3].

Ontology evaluation is needed to decide on the quality and content of our ontology by judging it against a reference framework and identifying what the ontology defines correctly, incorrectly or not at all. It is critical for the adoption and improvement of the ontology [6]. To provide high-quality ontology, evaluation techniques need to be continuously applied during the whole construction process as well as before the release and reuse of an ontology [3]. A high-quality ontology is easily explored and interpreted by its users. Furthermore it should be consistent, complete and interoperable for representing adequately the emergency knowledge. It guarantees that what is built meets the application requirements, whereas existing errors and omissions may lead to the inability of the application to work properly and not serve its intended purpose. A good ontology is more likely to be reused and will easily detect and actively neglect errors contained in the data and the reasoner is able to infer the right conclusions [7], [8]. In recent literature, many different approaches for ontology evaluation are suggested and explained. They are divided into quantitative and qualitative methods from subjective evaluations by experts to different tools and measurements which investigate different quality metrics of ontologies. These methods can be manual or automatic, evaluate the structure or the content of an ontology and dependent or independent of a specific tool. As each approach investigates different aspects, it is important to use several and complementary methods [9], [10].

In this paper a short overview of different approaches and methods suggested in related research is given before proposing the evaluation framework collocated for this 
evaluation which consists of ontology verification and validation. Verification relates to the correctness of the built ontology and especially investigates the structure, functionality and representation of the ontology with the help of different metrics and quality criteria. Validation relates to the question if the right ontology is built given the suggested application of the ontology. The contribution of this work is to propose an evaluation framework to evaluate the emergency situation ontology (ESO); and then evaluate and thereby refine the ESO over that framework so that it is able to fit for its intended task, i.e., generating recommendations in the emergency situations. The ESO has been verified for its structure using two tools namely OntoMetric and OOPS. The ontology is refined based on the results of both the tools. The ESO has been validated semantically using various competency questions to determine its applicability. The ESO has then been put to the said application and the task based evaluation has been carried out. It is found that ESO is able to serve its intended purpose after tuning it based on the results of evaluation [9], [11].

\section{OVERVIEW OF RESEARCH IN ONTOLOGY EVALUATION}

Many researchers have worked on ontology evaluation, thus different approaches, methods and tools are available, each developed for different purposes like the assessment of a newly developed ontology (developer centric evaluation) or ontology selection which finds the best suited ontology for a certain application in a set of ontologies (user centric evaluation) [7].

The declarative nature of ontologies and their use and extension outside a central control mechanism make ontology evaluation a difficult task. Even so there exists a large amount of methods and tools there is no standard approach for ontology evaluation yet as ontologies are semantic oriented and therefore always need individual assessment [12].

To achieve the best results and high quality ontology, one needs to choose from the available list of aspects of ontology to be evaluated; the right approach to evaluation; the right mix of criteria to be evaluated; and also the right tools to be used.

\section{A. Aspects}

Aspects include the vocabulary, syntax, structure, semantics, representation and context of the ontology, which are defined as follows [7], [12]

1) Vocabulary is the set of all names used in an ontology, whereby names are either URIs or literals. It evaluates how well a concept, instance or fact is named, comparing it to various data sources of the given domain.

2) Syntax and Structure deals with the structure and hierarchical relations that describe the ontology itself. It is evaluated with the help of predefined quality criteria.

3) Semantic is concerned with the content and meaning of the ontology.

4) Representation evaluates how adequately the semantic content is represented by the structure of the ontology.

5) Context takes the application for which the ontology is designed into account and evaluates the usefulness of the ontology for its purpose.

\section{B. Approaches}

The different known methods and techniques can be mainly assigned to four different kinds of approaches: Technology-based, quality-attribute based, data-driven and application driven evaluation.

1) Technology-based evaluation is concerned with the structural characteristics of an ontology. It investigates the syntax, consistency and formal semantics and thereby ensures the correctness and usability of the ontology. The approach cannot tell anything about the quality of the content and applicability of the ontology [2], [13].

- OOPS! is a web-based tool that is independent of any ontology development environment and acts as a pitfall scanner for identifying potential errors that may lead to inconsistencies and reasoning errors. It is accompanied by a catalogue of potential and common pitfalls [14].

2) The quality-based approach offers a quantitative evaluation which relies on a set of predefined metrics that measure individual quality attributes of an ontology. It is useful to detect formal and semantic inconsistencies, redundant terms, inconsistencies and missing definitions. Some of those quality metrics tend to be hard to measure and might need human experts to evaluate [12].

- The OntoClean methodology proposed by M. Fernández-López and A. Gómez-Pérez is based on philosophical notions like rigidity, unity, identity and dependence. It is used for detecting formal and semantic inconsistencies.

- OntoMetric suggests a set of measures to select the best ontology for a particular need by allowing the user to assess the suitability of an ontology for a given application [10].

- OntoQA is a metric-based approach for ontology quality analysis which offers five schema quality features and nine knowledge base quality features which investigate the quality of an ontology based on the instances it contains [15].

3) Data-driven evaluation: As the structural quality of an ontology only implies the usability of the ontology but not the suitability for the given application, this approach concentrates on the usability of an ontology considering its future application. In this approach the ontology is compared to data sources about the covered domain. Case studies can be used or the concepts and relations in the ontology can be compared to text documents to check if the ontology has enough concepts to represent the real world knowledge. This evaluation approach attempts to analyze how adequate an ontology covers the domain but is not applicable to determine the correctness or clarity of the ontology [9], [16].

4) Application-based evaluation

In the application-based approach the ontology is used within its specific application. Thereby its content is evaluated concerning its usability and correctness. This approach is not suited for a general evaluation approach, because every ontology must be evaluated individually depending on the application context [9]. 


\section{Criteria}

Various criteria have been proposed in literature to evaluate the quality of ontology [7], [12], [17]. Some of these criteria are quantifiable but some are not, so a human will be required to assess these. One needs to identify the attributes to assess the quality of his ontology.

1) Consistency: Enables the reasoner to infer knowledge and interpret the ontology, whether it is according to specification with no contradictions or conflicting information. For this purpose the Pellet reasoner developed by the Mindswap laboratory at Maryland University is used. Obtained inconsistencies need to be debugged and repaired.

2) Completeness: Assures that all important information is included in the ontology. Completeness can be evaluated regarding the language, the domain and the application requirements.

3) Accuracy: Determines if the expert knowledge about the domain approves of the contained knowledge in the ontology, i.e., distance between real world and conceptualization. This is assessed by interviewing experts.

4) Conciseness: To make sure no irrelevant information is included which makes the ontology unnecessarily large and increases computational resources. Redundancies should be removed.

5) Correctness: Assures that the ontology depicts the correct modeling of the real world. This is assessed by precision.

6) Computational Efficiency: It is a measure of how easy and successful can reasoners process the ontology

7) Adaptability: Refers to the expandability, sensitiveness, extendibility, interoperability or the flexibility of the ontology.

8) Clarity: Refers to the understandability and the transparency of the ontology.

\section{Tools}

Various tools have been developed to support the task of ontology evaluation, each concerned with different aspects of evaluation. There exist tools for checking the consistency, the structure or modeling mistakes of the ontology [13]. Various available tools include: ODEClean, ODEval, AEON, Eyeball, Moki, XD-Analyzer, OQuaRE, OntoCheck, OntoQA, OntoClean, OntoMetric, ACTiveRank, OOPS!, ODEval, oQual.

The people in the field have proposed various ways of evaluating the domain ontology. Even so a lot of research has been done in this area, no ideal solution has been found yet. Most of the approaches are oriented to a few dimensions and most use only one aspect. Regarding tools, most have been developed as plugins for desktop applications except a few. They calculate the value of metrics but do not map them to the criteria under consideration. Each approach has its own limitations and therefore a combination of several approaches is selected and introduced in the following section to evaluate the emergency situation ontology.

\section{Proposed EVAluation FramewORK FOR ASSESSING INDIVIDUAL ONTOLOGIES}

The approach of ontology evaluation proposed in this paper follows the goal of assessing an individual ontology by itself, especially adapted to evaluate the emergency situation ontology introduced beforehand. There exists no standard approach for evaluation as each ontology deals with a different domain and some ambiguity is always involved. The evaluation framework introduced in this paper is a combination of several already existing and reliable evaluation methods and tools. It is divided into the different layers of the ontology: lexical, structural/ functional, semantic and application. This simplifies the evaluation of the ontology by ensuring that each characteristic of the ontology is evaluated separately instead of evaluating the ontology as a whole [12]. The first two layers are concerned with the verification of the ontology, whereas the remaining two layers deal with the validation of the ontology.

\section{A. Lexical Layer}

Evaluating the lexical layer is necessary to improve the understandability and reusability for other users using the ontology. It assesses if classes, properties and individuals are named consistently and meaningful and if the amount and quality of annotations is sufficient. Thereby it ensures a sufficient knowledge representation, making the ontology easier to reuse and compare to other ontologies [12], [18].

\section{B. Structural / Functional Layer}

The evaluation of the structural and functional layer is important for assessing the hierarchical and structural relations between concepts, as otherwise major errors and reasoning problems can occur which make the whole ontology useless. In this layer the consistency and interoperability is investigated. Completeness and Conciseness is evaluated by checking individually each class, property and instance for incompleteness, inconsistence or redundancy errors as proposed by Gomez-Perez seen in table $1[6]$.

TABLE I: COMMON ERRORS DEFINED BY GOMEZ-PEREZ

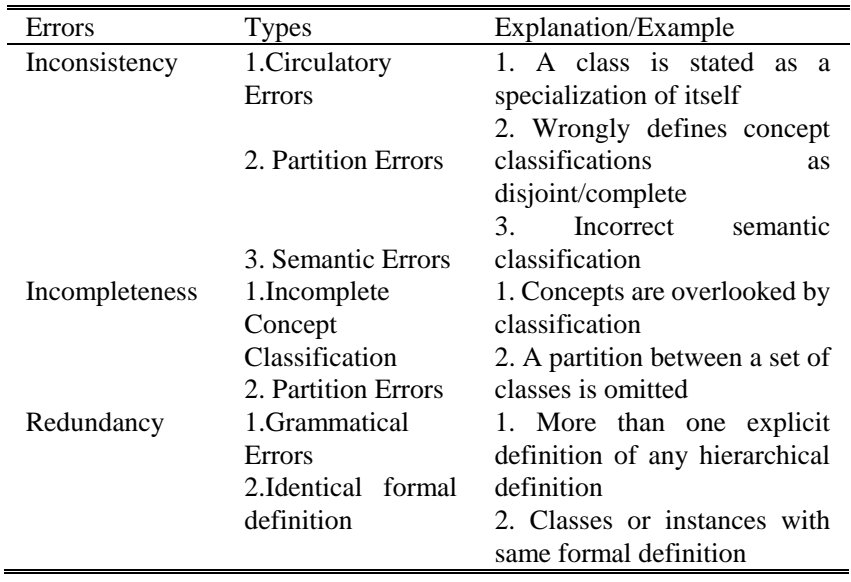

The quantitative evaluation of structured layer is done using metric based approach with the OntoMetric tool and the qualitative evaluation is done using criteria based approach with the OOPS tool.

The OntoMetric evaluation tool offers metrics which evaluate the ontology based on the knowledge represented in the instances and thus measures the quality and main characteristics of the ontology. The proposed quality criteria 
consist of schema metrics which evaluate the design of the ontology and instance metrics which evaluate how instances are organized in the ontology. OOPS! is a platform independent online tool which detects errors in ontologies according to a pitfall catalogue currently containing 41 errors It helps ontology developers to find common pitfalls during ontology verification. Nonetheless the tool is not able to repair the found inconsistencies; this must be done by the evaluator. OOPS! is a helpful and easily used tool for supporting ontology evaluation [13]. Table II lists some of the common pitfalls along with their description which we wish should not occur in any ontology.

TABLE II: SOME COMMON PITFALLS OF OOPS

\begin{tabular}{|c|c|}
\hline \multicolumn{2}{|c|}{ Consistency } \\
\hline P05 & Define incorrect inverse relationship \\
\hline P06 & Involve cycles in hierarchy \\
\hline $\mathrm{P} 07$ & Merging dissimilar concepts in same class \\
\hline P19 & Swapping intersection and union \\
\hline $\mathrm{P} 02$ & Using recursive definition \\
\hline \multicolumn{2}{|c|}{ Conciseness } \\
\hline $\mathrm{P} 02$ & Creating Class Synonyms \\
\hline $\mathrm{P} 03$ & $\begin{array}{l}\text { Creating "is" relationship place of "rdfs:subClassOf," "rdf:type" } \\
\text { or "owl:sameAs" }\end{array}$ \\
\hline $\mathrm{P} 21$ & Using a miscellaneous class \\
\hline \multicolumn{2}{|c|}{ Completeness } \\
\hline P04 & Creating unconnected ontology elements \\
\hline P10 & Missing disjointness \\
\hline P11 & Missing domain or range in properties \\
\hline $\mathrm{P} 12$ & Missing equivalent properties \\
\hline $\mathrm{P} 13$ & Inverse relationships not explicitly declared \\
\hline \multicolumn{2}{|c|}{ Correctness } \\
\hline P10 & Missing disjointness \\
\hline \multicolumn{2}{|c|}{ Clarity } \\
\hline P08 & Missing Annotations \\
\hline $\mathrm{P} 22$ & Using different naming conventions in the ontology \\
\hline
\end{tabular}

The combined use of these two tools offer a wide range of different evaluation aspects and thus the quality and characteristics are assessed on many different levels.

\section{Semantic Layer}

The semantic layer assesses if all necessary data is included in the knowledge base and if the ontology represents the domain sufficiently. It evaluates the semantic completeness, accuracy, conciseness and correctness of the ontology. For this purpose domain experts are involved in the evaluation.

\section{1) Competency questions}

For the ontology validation, competency questions as a qualitative measure is the most effective and reliable way to check if all important information are included in the ontology. The ontology requirements are formulated as natural language expressions for which the correct answers are provided. By translating the questions into formal SPARQL queries and evaluating them it can be simply concluded which information are missing in the ontology and need to be added.

It needs to be remembered that competency questions are not an exhaustive qualitative measurement, and that even if all queries can be answered correctly the ontology may not be complete [19].

\section{2) Expert interviews}

For assessing the completeness of the ontology domain experts are involved in the evaluation. Each expert receives a questionnaire especially developed for the domain and application of the ontology containing several questions where they can value the completeness, accuracy and conciseness of the classes and properties contained in the ontology. The experts are able to rate each answer and give additional remarks about the ontology to specify missing classes and properties or state redundancies.

\section{Application Layer}

The fitness of the ontology regarding the intended application can be assessed by using test cases and by actual usage of the ontology within the application. This is the last step of the evaluation and can only be done in the last stage of ontology development when a finished ontology is available [16].

\section{EVAlUATION OF EMERGENCY SitUATION ONTOLOGY}

In this section the evaluation of the emergency situation ontology according to the evaluation framework is discussed. We developed this ontology as part of a project to prepare an advisory system for emergency management. This advisory system is supposed to generate recommendations using the mixed approach of rule based reasoning and case based reasoning to provide an automatic and effective response for a fast reaction to any emergency situation. The aim is to minimize the loss of life and property, thus the ontology serves as a knowledge base that stores information, recommendations and expert advice about emergency situations and the decisions taken for an effective and immediate emergency response [5], [20].

\section{A. Lexical Layer}

Human based approach is used in this layer to assess ESO. Evaluating the lexical layer indicates that the naming of the ontology is not consistent. The naming has been adapted to the CamelCase style and unclear naming is improved, making it easier to understand and reuse the ontology. Furthermore annotations are added in the ontology to offer natural language documentation and make the ontology easier to understand for humans.

\section{B. Structural / Functional Layer}

The assessment of the structural and functional layer starts with investigating the logical consistency of the ontology. The ESO has been debugged with the use of the Pellet reasoner and found inconsistencies have been corrected. The interoperability of the ontology is guaranteed by using OWL2 to preserve codification standards and present an interoperable knowledge platform.

Evaluation of the consistency and completeness of the taxonomy according to the errors described in Table I revealed that mainly incomplete concept classification and partition errors concerning the completeness have been made while developing the ontology. For example has the developer created a class 'ServiceProvider' with several subclasses but omit that these classes are disjoint. The same holds for the class 'Region' divided into 
'UnpopulatedRegion' and 'PopulatedRegion'. It needs to be stated that these classes form an exhaustive subclass partition, e.g. these classes build the complete subset of the class 'Region'. Evaluating the conciseness of the emergency situation ontology mainly redundancy errors concerning an identical formal definition of some classes have been found. These are cleared by merging the concerned classes into one class. For example the classes 'Action' and 'ReactionsOnEmergency' have the same purpose and formal description and thus are merged into one single class.

The obtained results of the manual evaluation of the ontology are supported by the results of the OOPS! pitfall scanner [21] seen in Fig. 1. The online tool reliably detects most errors found in the ontology, shows where to find them and suggests improvements. Nonetheless the modifications of the ontology needs to be done manually and additional evaluation is needed as some errors, especially if semantics are involved, are not detected. For example the tool was not able to detect that the subClassOf relation is falsely used instead of creating a 'is part of' relation, e.g. the class ' $C$ ity' is a subclass of 'Country'. After correcting the observed errors the pitfall scanner is run again to ensure all errors are corrected and no new ones are detected.

\begin{tabular}{|ll|}
\hline Results for P04: Creating unconnected ontology elements. & 3 cases $\mid$ Minor \\
\hline Results for P07: Merging different concepts in the same class. & 3 cases $\mid$ Minor \\
\hline Results for P08: Missing annotations. & 313 cases $\mid$ Minor \\
\hline Results for P11: Missing domain or range in properties. & 1 case $\mid$ Important 0 \\
\hline Results for P13: Inverse relationships not explicitly declared. & 13 cases $\mid$ Minor \\
\hline Results for P22: Using different naming conventions in the ontology. & ontology $\mid$ Minor \\
\hline
\end{tabular}

Fig. 1. Results of OOPS! pitfall scanner.

The pitfalls in error were corrected and the result is shown in Fig. 2 below:

\begin{tabular}{|ll|}
\hline Results for P04: Creating unconnected ontology elements. & 1 cases $\mid$ Minor \\
\hline Results for P07: Merging different concepts in the same class. & 1 cases $\mid$ Minor \\
\hline Results for P08: Missing annotations. & 2 cases $\mid$ Minor \\
\hline Results for P13: Inverse relationships not explicitly declared. & 3 cases $\mid$ Minor \\
\hline Results for P22: Using different naming conventions in the ontology. & ontology ${ }^{*} \mid$ Minor \\
\hline
\end{tabular}

Fig. 2. Results of OOPS! pitfall scanner after refinement.

Here we describe the metrics of OntoMetric chosen to determine the characteristics of ESO and the results discussed:

\section{Schema Metrics:}

1) Relationship Richness (RR) is calculated as the ratio between the number of non-inheritance relationships divided by the overall number of relationships, thus it indicates the diversity of relationships in the ontology. The result is a number between 0 and 1, where a high number indicates that the variety of complex relationships is high and the ontology does not only consist of simple inheritance relationships. About the emergency situation ontology can be concluded that already a vast amount of diverse relations are existing, nonetheless the result can be improved.

2) Inheritance Richness (IR) is defined as the average number of subclasses per class. It describes how classes are distributed across the different layers of the ontology inheritance tree and thus distinguishes shallow from deep ontologies. The relatively low result indicates that the emergency situation ontology represents its domain very detailed. A high result in contrast would indicate that an ontology depicts a wide range of general knowledge without a lot of details.

3) Attribute Richness (AR) is described as the average number of attributes per class, thus it assesses the quality of ontology design and the amount of information instances contain. The more attributes the ontology contains the more knowledge it contains, thus a high result is preferable.

\section{Knowledge-base Metrics:}

1) The Class Richness (CR) calculates the number of classes that contain individuals, thus it indicates how well the knowledge stored in the ontology as instances is distributed. The low result for the emergency situation ontology shows that the ontology does not contain enough knowledge to represent all concepts in the ontology. As the development of the emergency situation ontology is not finished yet, it indicates that the ontology is not populated yet and more knowledge needs to be added.

2) Class Connectivity (CC) calculates the overall number of relationships the instances of the class have with instances of other classes and thus indicates which classes are central in the ontology. In the case of the emergency situation ontology a special focus lies on the different emergency situations and required services and resources, which illustrate the importance of these areas. The results calculating the metrics are seen in Table III.

TABLE III: PROPERTIES OF ESO AND RESULTS OF ONTO METRIC

\begin{tabular}{llllllll}
\hline \hline Classes & Relations & Instances & RR & IR & AR & CR & CC \\
\hline 228 & 266 & 120 & 0.74 & 1.73 & 2.7 & 0.135 & 11 \\
\hline \hline
\end{tabular}

\section{Semantic Layer}

By providing a set of competency questions for the validation purpose, the completeness of the ontology is evaluated. In total a set of 36 competency questions covering the domain of emergency response is provided in natural language and is translated into SPARQL queries. Each query is run on the ontology to test if all requirements can be met and the correct answers can be inferred. For those queries that fail to run, the missing concepts or relations are added in the ontology. Nonetheless the completeness of the ontology can never be proved and constant enhancement of the emergency situation ontology is needed. Table 4 provides two sample competency questions with the results when run on ESO.

In addition to the competency questions, expert interviews have been conducted to evaluate the completeness, correctness and conciseness of the ontology. A questionnaire containing 20 questions concerning the importance and clarity of each concept inside the ontology has been developed, so that domain experts in the field of emergency management are able to rate the ontology and give additional 
feedback. By means of the received answers we were able to conclude which concepts are redundant, which are still missing and on which concepts the main focus lies.

TABLE IV: EXAMPLE OF COMPETENCY QUESTIONS AND SPARQL QUERIES 1. What are the major causes of the Earthquake?

SELECT ?Causes ?DefinitionWHERE \{es:Earthquake

rdfs:subClassOf ?restriction.?restriction owl:onProperty

es:caused_by.?restriction owl:someValuesFrom |

owl:allValuesFrom ?Causes .optional\{?Causes rdfs:comment ?Definition\}\}

Causes Definition

ToctonicPlate The two sub-layers of the earth's crust (lithosphere) that move, float, and sometimes fracture and whose interaction cause continental driff, earthquakes, volcanoes, mourtains, and oceanic treeches.

StismicWres A wave of energy that is zeneratad byan earthquake or other eart vibration and that travels within the earth or along its surface

2. Display the actions that were taken during the J\&K Earthquake

SELECT ?Actions ?Resource ?Numberofneededresources WHERE

$\{$ ? earthquake rdf:type es:GroundShaking. ?earthquake es:has_location

es:Darmstadt ?earthquake es:needs_action?Actions.?Actions

es:needs_recource ?Resource.?Resource

es:number_of_needed_resources ?Numberofneededresources.

\begin{tabular}{lll} 
Actions & Resources & NumberofNeededResources \\
ClearingWork & SpecialVehicles & 9 \\
SupplyGoodsDelivery & SupplyGoodSet & 1500 \\
MedicalDelivery & MedicalSet & 300 \\
Evacuationplan & TransportVehicle & 50 \\
Observation & ObservationDrones & 5 \\
\hline
\end{tabular}

\section{Application Layer}

The effectiveness of ESO has been assessed by putting it to the real application it was designed to work for as a knowledge base, i.e., decision support in case of an emergency and specifically an earthquake. As soon as an earthquake instance is being input to the system, it does preprocessing and fetches the population of that location and other important parameters as intermediate output from the ESO. The recommendation engine then advices the course of action to be taken based on available resources as instances in the ESO. It is found that the ESO is working as expected.

\section{DISCUSSION}

The evaluation of the emergency situation ontology indicates that the ontology is well-designed and suitable for its application. Only minor changes and adaptations regarding the lexical and structural layer are made. The validation of the ontology revealed that most concepts relevant for the application are already included in the ontology. Nonetheless even after the evaluation it cannot be stated that the ontology is fully complete as it needs constant improvement and adaptation while active usage.

Disadvantages of the evaluation framework lie in the subjectivity of the ontology evaluation. The evaluator has to decide on the criteria relevant for the evaluation, evolves the competency questions and has to make decisions based on the evaluation results over each metric. But as good science should exclude subjectivity, it is advisable that more than one person performs the evaluation. Furthermore experts should be included in the evaluation.

\section{CONCLUSION}

Ontology evaluation is a main task of ontology engineering that takes a lot of effort and thought-process as each ontology needs an individual approach for evaluation adapted to the intended application of the ontology. There exists no possibility for a single approach to fit for every application context, so instead a framework is proposed in this paper which considers the different aspects and quality criteria that need to be evaluated in each evaluation layer considering the different ontology construction phases.

The evaluation of the emergency situation ontology illustrates that the evaluation process is limited. More expert reports and opinions need to be taken into account for further evaluation and the actual assessment of the ontology can only be done by active usage of the ontology after its inclusion into the emergency response system.

Furthermore evaluating the emergency situation ontology demonstrated that most probable no automatic method will ever be enough to perform a complete ontology evaluation. Instead individual expert advice is needed for a satisfactory result. Nonetheless automatic and semi-automatic methods can be further developed and improved as the application of these methods renders the evaluation easier, faster and less subjective and turns out to be sufficient for ontology verification.

\section{ACKNOWLEDGMENT}

V. Meyer thanks DAAD Rise Worldwide Internship Program for offering an opportunity to work in India.

\section{REFERENCES}

[1] T. R. Gruber, "Towards principles for the design of ontologies used for knowledge sharing," International Journal of Human-Computer Studies, vol. 43, no. 5/6, pp. 907-928, 1995.

[2] N. Casellas, "Ontology evaluation through usability measures," On the Move to Meaningful Internet Systems, Springer Berlin/Heidelberg, vol. 5872.

[3] S. Jain, "Intelligent decision support for unconventional emergencies," Exploring Intelligent Decision Support Systems. Studies in Computational Intelligence, 2018, vol. 764, Springer, ISBN: 978-3-319-74001-0.

[4] S. Mishra and S. Jain, "A study of various approaches and tools on ontology," in Proc. 2015 IEEE International Conference on Computational Intelligence \& Communication Technology (CICT), Feb 13-14, 2015, pp. 57-61.

[5] S. Mishra, S. Malik, N. K. Jain, and S. Jain, "A realist framework for ontologies and the semantic web," Procedia Computer Science, vol. 70, pp. 483-490, Dec 2015.

[6] A. Gómez-Pérez, "Evaluation of ontologies," International Journal of Intelligent Systems, John Wiley \& Sons, Inc., pp. 391-409, 2001.

[7] D. Vrandecic, "Ontology evaluation," Handbook on Ontologies, Springer Berlin/ Heidelberg, 2009, pp. 293-313.

[8] H. Hlomani and D. Stacey, "Approaches, methods, metrics, measures, and subjectivity in ontology evaluation: A survey," Semantic Web Journal, 2014.

[9] G. Bilgin, I. Dikmen, and M. TalatBirgonul, "Ontology evaluation: An example of delay analysis," presented at Creative Construction Conference 2014, 2014.

[10] J. Hartmann, P. Spyns, A. Giboin, D. Maynard, R. Cuel, M. C. Suárez-Figueroa, and Y. Sure, "D1 2.3 methods for ontology evaluation," EU-IST Network of Excellence IST-2004-507482 KWEB Deliverable D, vol. 1, 2005.

[11] Y. Sure, "Methodology, tools and case studies for ontology based knowledge management," Ph.D. dissertation, Fakultätfür Wirtschaftswissenschaften der Universität Friedrich zu Karlsruhe, 2003. 
[12] J. Pak and L. Zhou, "A framework for ontology evaluation," in Workshop on E-Business: Springer Berlin/Heidelberg, 2009, pp. $10-18$.

[13] A. Gangemi, C. Catenacci, M. Ciaramita, and J. Lehmann, "Modelling ontology evaluation and validation," presented at the 3rd European Semantic Web Conference, Budva, Montenegro, 2006.

[14] M. Poveda-Villalón, M. C. Suárez-Figueroa, and A. Gómez-Pérez, "Validating ontologies with OOPS!" Knowledge Engineering and Knowledge Management, pp. 267-281, 2012.

[15] S. Tartir, I. B. Arpinar, and A. P. Sheth, "Ontological evaluation and validation," Theory and Applications of Ontology: Computer Applications, Springer, Dordrecht, 2010.

[16] A. Zouaq and R. Nkambou, "Evaluating the generation of domain ontologies in the knowledge puzzle project," Knowledge and Data Engineering, vol. 21, no. 11, 2009.

[17] A. Gómez-Pérez, "Ontology evaluation," Handbook on Ontologies, Springer Berlin/Heidelberg, 2004, chapter 13, pp. 251-274.

[18] M. Horridge, "A practical guide to Building OWL ontologies using Protégé 4 and CO-ODE tools," The University of Manchester, March 24, 2011.

[19] M. Gruninger and M. S. Fox, "Methodology for the design and evaluation of ontologies," in Proc. Workshop on Basic Ontological Issues in Knowledge Sharing, Montreal, Canada, 1995.

[20] C. Gupta, A. Bhardwaj, S. Tiwari, and S. Jain, "A semantic web portal for unconventional emergencies," presented at IEEE International Conference on Computing, Communication and Networking, 2017.
[21] Linkeddata. [Online]. Available: http://oops.linkeddata.es/

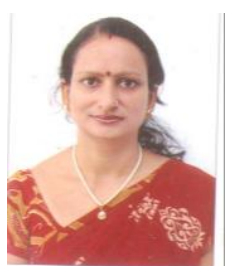

Sarika Jain graduated from Kurukshetra University (India) in 1998 and did her masters from Jawaharlal Nehru University (India) in 2001. Her doctorate is in the field of knowledge representation in artificial intelligence which was awarded in 2011. She has served in the field of education for over 17 years and is currently serving at National Institute of Technology, Kurukshetra. Her current research interests are in the area of intelligent systems, including knowledge representation, ontological engineering and semantic web technologies. She is a member of IEEE and ACM and a Life Member of Computer Society of India.

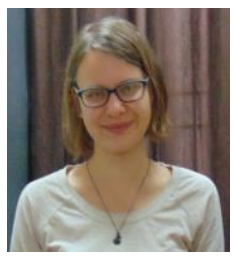

Valerie Meyer was born in Adenau, Germany on Feb. 17,1995 . She is currently studying bachlore of sciene in cognitive science at the University of Osnabrueck in Germany. This writing is part of my internship at the National Institute of Technology Kurukshetra, India. 